\title{
Prevalence of Pulmonary Tuberculosis among Rohingya Refugees in Kutupalong Camp, Bangladesh
}

Jasmine $\mathrm{R}^{1}$, Khan $\mathrm{AA}^{2}$, Kamal AHMKM ${ }^{3}$, Karim ATMR ${ }^{4}$, Haque $\mathrm{MM}^{5}$

DOI: https://doi.org/10.3329/jafmc.v14i2.45909

\begin{abstract}
Introduction: An estimated over seventy thousands Rohingya refugees living in Bangladesh since August 2017. World Health Organization (WHO) warned that there could be an outbreak of infectious diseases like tuberculosis (TB) among the Rohingya that would also be a threat to the local people.
\end{abstract}

Objectives: To find out the prevalence of TB among Rohingya refugees in Kutupalong camp, Ukhiya.

Materials and Methods: This cross-sectional observational study was conducted at the field medical laboratory of Bangladesh Army in the refugee camp Kutupalong, Ukhiya from September to December, 2017. Total 3579 patients reported during this time thosehad cough for more than 2 weeks were selected as study subjects. The Ziehl Neelsen stained smears were prepared from the sputum samples and examined only under microscope for acid fact bacilli (AFB). All AFB positive cases were tested by molecular technique, Genexpert for drug resistance and screened by immunochromatographic test(ICT) for HIV.

Results: Out of 3579 reported cases $55(1.54 \%)$ were AFB positive. Among 55 positive cases $27(49.09 \%)$ were female and $28(50.90 \%)$ were male.There was no HIV casefound among 55 AFB positive patients but 01 case was found positive as multi drug resistant TB (MDR-TB).

Conclusion: This study showed prevalence of TB is quite high in Rohingya population due to malnutrition, unhealthy living condition and general lack of awareness. Directly Observed Treatment Short Course (DOTS) had already started to eradicate TB which will definitely prevent contagiousness to local people in Bangladesh.

Key- words: Mycobacterium tuberculosis, DOTS, Pulmonary tuberculosis, MDR-TB.

\section{Introduction}

According to WHO global tuberculosis report 2016, Bangladesh is one of the world's 30 high TB burden countries with annual occurrence of 362,000 new cases. About 73, 000 people die annually due to TB. The organization also believes one billion people globally will become infected and 36 million will die of TB by 2020 , unless control measures are strengthened ${ }^{1}$. A total of 33,131 registered Rohingya refugees are living in two registered camps in Cox's Bazar, and up to 80,000 additional refugees are housed in nearby makeshift camps. For a huge population of Rohingyas in Kutupalong refugee camp, Ukhiya, they are suffering from many health problems specially TB, respiratory tract diseases, skin diseases and many others ${ }^{2}$.

TB is a disease caused by inhalation of aerosolized organism M. tuberculosis, most commonly is transmitted from "smearpositive" casesby coughs, sneezes, talks and its initial site of infection is the lung. Crowded conditions, malnutrition and generally poor quality of life like refugee camps contribute to disease spread ${ }^{3}$. If a person has confirmed TB or is suspected of having $T B$, the best way to stop transmission is through immediate isolation ${ }^{4}$. Therapy should begin immediately. Contagiousness declines rapidly after a standard treatment regimen has begun, provided the patient adheres to the course of therapy ${ }^{4,5}$. Resistance to INH and other anti TB drugs is being seen in Southeast Asia and Latin America ${ }^{6}$. Strains of M. tuberculosis resistant to multiple drugs have emerged, primarily in AIDS patients, or close contact with an individual who is infected with MDR-TB. Rakhaine state Myanmar lacks a proper health infrastructure and a vaccination program, which initially puts the Rohingya population at an increased risk of developing TB from their native country and upon migration to Bangladesh ${ }^{7}$. To find out the prevalence of TB among Rohingya refugees in Kutupalong camp this study was planned.

\section{Materials and Methods}

This cross-sectional observational study was carried out at the field medical laboratory in the refugee camp Kutupalong, Ukhiya, Cox's Bazar from September to December, 2017. Total 3579 patients reported during this time those had coughed for more than 2 weeks were included as study subjects. The numbers also included the known cases of TB who had been being under treatment in Myanmar. The Ziehl Neelsen stained smears were prepared from the sputum samples and examined only under microscope for AFB. All TB positive cases were screened by ICT test for HIV. The patients who reported

1. Lt Col Rezina Jasmine, MBBS, DCP, MCPS, FCPS, Classified Specialist in Pathology, AFIP, Dhaka (E-mail: rezina.jasmine@gmail. com) 2. Brig Gen Arif Ahmed Khan, MBBS, MCPS, FCPS, Classified Specialist in Pathology \& Deputy Commandant, AFIP, Dhaka 3. Col A H M Kazi Mostofa Kamal, MBBS, FCPS, Classified Specialist in Psychiatry, CMH, Dhaka 4. Lt Col A T M Rezaul Karim, MBBS, DDV, MCPS, FCPS, Associate Professor of Dermatology, AFMC, Dhaka 5. Col Md Monirul Haque, MBBS, DCP, MCPS, FCPS Classified Specialist in Pathology, AFIP, Dhaka. 
with cough not more than two weeks and were found as infections by other bacteria like Streptococcus spp, Klebsiella spp, Staphylococcus spp and others were excluded. The positive cases were tested by molecular technique, Genexpert for MDR-TB at Cox's bazaar town which is far away from the refugee camp. There were no provisions for $M$. tuberculosis culture in the field laboratory, so bacterial cultures of the sputum couldn't be done.

\section{Results}

In this study, out of 3579 , patients 55 cases were identified as TB patientsand the overall prevalence was $1.54 \%$ (Figure-1). Among the 55 AFB positive cases $28(50.90 \%$ ) were male and $27(49.09 \%)$ were female and ratio was 1.03:1. The patients were divided into 8 groups according to the age differences of 10 years as Group I( $<10$ years) to Group VIII ( $70-79$ years). Among male patients most common AFB positive cases were in Group VII followed by Group III but among female patients most common AFB positive cases were in the age Group VI followed by Group III and Group V (Table-I). Only 01 patient was found MDR-TB which was detected by Genexpert but no HIV positive case found among 55 AFB positive patients.

Table-I: Distribution of TB patients by age group and sex

\begin{tabular}{|l|c|c|c|}
\hline \multirow{2}{*}{ Groups according to age difference } & \multicolumn{2}{|c|}{ Sex } & \multirow{2}{*}{ Total } \\
\cline { 2 - 3 } & Male & Female & \\
\hline Group I (<10 years) & 0 & 0 & 0 \\
\hline Group II (10-19 years) & 0 & 03 & 03 \\
\hline Group III(20-29 years) & 06 & 05 & 11 \\
\hline Group IV (30-39 years) & 02 & 04 & 06 \\
\hline Group V (40-49 years) & 02 & 05 & 07 \\
\hline Group VI (50-59 years) & 05 & 07 & 12 \\
\hline Group VII (60-69 years) & 10 & 03 & 13 \\
\hline Group VIII (70-79 years) & 03 & 00 & 03 \\
\hline Total & $\mathbf{2 8}$ & $\mathbf{2 7}$ & $\mathbf{5 5}$ \\
\hline
\end{tabular}

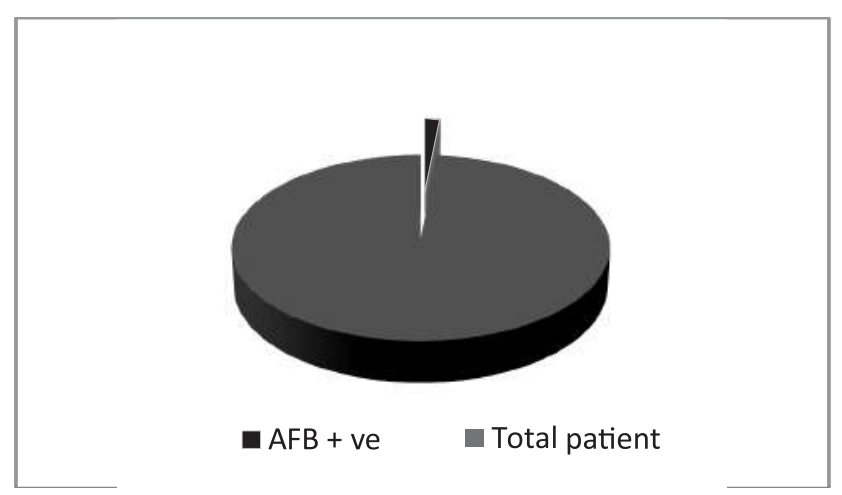

Figure-1: Prevalence of TB cases

\section{Discussion}

Of the infectious diseases prevalent in refugee camps, Tuberculosis (TB) is a major public health concern amongst the Rohingya population primarily due to the high risk of transmission, rapid progression of the disease, and the cost of burden due to poor living conditions ${ }^{8}$.
In this study, out of 3579 reported cases, 55 were AFB positive. Among them, 27(49.09\%) were female and 28(50.90\%) were male. All TB positive cases were screened for HIV but there was no HIV positive case found. As per reports, the rate of tuberculosis notification in Rakhine state in 2016 was $231 / 100,000$ compared to the overall rate of $217 / 100,000$ in Myanmar. HIV among TB cases was 3\% (national range 9.6\%). The rate of incidence of TB in Myanmar is 365/100,000 (higher than Bangladesh rate of 225). Extrapolating this rate and using a prevalence rate of 500/100,000, one can expect to have about 4000 TB patients among the displaced persons now in the camps ${ }^{2}$. With a prevalence of 525 cases per 100,000 population it was estimatedthat approximately between 5-15 cases of active TB in each of test populations for somewhere around 20-30 total cases TB in Rohingya of active TB7.

In the United States, 6 cases of XDR-TB were reported in 2011, bringing the total to 12 since 2008. Of the 12 XDRTB cases, 11 were in foreign-born persons ${ }^{9}$. Because of the threat of MDR-TB and XDR-TB, it is important for laboratories to identify Mycobacterium spp. rapidly and perform antimicrobial susceptibility testing so that appropriate therapy can be administered as quickly as possible ${ }^{10,11}$. Rohingyas vulnerable population has low vaccination coverage, high prevalence of malnutrition and exposure to poor living conditions. As a result, vaccination-preventable diseases are prevalent among this population ${ }^{12}$. Directly Observed Treatment Short Course (DOTS)had already started to treat TB casesas part of the Government's program ${ }^{13}$. $B C G$ vaccination was recommended after birth to avoid life threatening TB diseases. Most of the refugees are located along the Teknaf-Cox's Bazar highway, a coastal area dependent upon tourism and difficult to prevent intermingling of Rohingya refugees with the Bangladeshi citizens ${ }^{13}$.

\section{Conclusion}

Pulmonary tuberculosis (TB) is the most common and infectious form of TB and among the greatest health concerns during immigration.Prevalence of TB is much higher among Rohingya refugees than local Bangladeshis and this vulnerable population has low vaccination coverage, high prevalence of malnutrition and exposure to poor living conditions. Special measures should be taken to prevent spread of TB to local peoples.

\section{References}

1. Regional Response Plan for TB-HIV-2017-2021. New Delhi: World Health Organization, Regional Office for South-East Asia; 2017. Licence: CC BY-NC-SA 3.0 IGO.

2. United Nations International Children's Fund (UNICEF). Rohingya Crisis. UNICEF; 2016 Apr [cited 2018 Jul 8]. https://www.unicef.org/ bangladesh/en/rohingya-refugee-crisis.

3. Levinson W. Review of Medical Microbiology and Immunology. 13th ed. New York: McGraw-Hill 2015; Chapter 21:180-9. 
4. Fares A. Seasonality of Tuberculosis. J Glob Infect Dis 2011; 3(1):46-55.

5. Isselbacher, Braunwald, Wilson, Martin, Fauci, Kasper. Tuberculosis. In: Harrison's Principles of Internal Medicine. 19th ed. New York; McGraw-Hill: 5 December 2017.

6. World Health Organization (WHO). WHO treatment guidelines for drug resistant tuberculosis 2016 update. Available from http://apps. who.int/iris/handle/10665/250125.

7. World Health Organization (WHO). The use of bedaquiline in the treatment of multidrug-resistant tuberculosis. Interim policy guidance. Geneva: WHO 2013. https:// apps.who.int/iris /bitstream /10665 /84879/1/9789241505482_eng.pdf, accessed 13 May 2016.

8. Perrelle J, Smith T, Mehta S. TB in Rohingya Refugee Populations: Opportunities for Health Stabilization through Cash Transfer and Monitoring. May 4th, 2018. https://www.researchgate.net/ publications/328346325.

9. World Health Organization (WHO). Guidelines for the programmatic management of drug-resistant tuberculosis. Geneva:
WHO 2011. Available at https://whqlibdoc. who. int/ publications/ 2011/9789241501583_eng.pdf, accessed 13 May 2016.

10. Treatment of Tuberculosis, American Thoracic Society, CDC and Infectious Diseases Society of America. Am J RespirCrit Care Med 2003; 52(RR11);1-77.

11. Das AC. Epidemic situation of tuberculosis in Bangladesh: An overview. South East Asia Journal of Public Health 2016; 6(2):61-2.

12. Redpath N. The Impact of Vaccination Programs on Forcibly Displaced Rohingya Children to Reduce Rates of Communicable Infections Within Bangladesh. MCHC 992.July 22, 2018.Available fromhttps:// pdfs. semanticscholar. org/3e8b/ 7399246ed66ec26dad11a158deb45ee15d45. pdf.

13. Alam N, Kenny B, Maguire JE et al. Field epidemiology in action: An Australian perspective of epidemic response to the Rohingya health emergencies in Cox's Bazar, Bangladesh. Global Biosecurity 2019; 1(1):119-22. 